\title{
TWO-SIDED MULTIPLICATION OPERATORS ON THE SPACE OF REGULAR OPERATORS
}

\author{
JIN XI CHEN AND ANTON R. SCHEP
}

\begin{abstract}
Let $W, X, Y$ and $Z$ be Dedekind complete Riesz spaces. For $A \in$ $L^{r}(Y, Z)$ and $B \in L^{r}(W, X)$ let $M_{A, B}$ be the two-sided multiplication operator from $L^{r}(X, Y)$ into $L^{r}(W, Z)$ defined by $M_{A, B}(T)=A T B$. We show that for every $0 \leq A_{0} \in L_{n}^{r}(Y, Z),\left|M_{A_{0}, B}\right|(T)=M_{A_{0}, B \mid}(T)$ holds for all $B \in L^{r}(W, X)$ and all $T \in L_{n}^{r}(X, Y)$. Furthermore, if $W, X, Y$ and $Z$ are Dedekind complete Banach lattices such that $X$ and $Y$ have order continuous norms, then $\left|M_{A, B}\right|=$ $M_{|A|,|B|}$ for all $A \in L^{r}(Y, Z)$ and all $B \in L^{r}(W, X)$. Our results generalize the related results of Synnatzschke and Wickstead, respectively.
\end{abstract}

\section{INTRODUCTION}

For an algebra $\mathcal{A}$ an operator of the form $T \mapsto \sum_{i=1}^{n} A_{i} T B_{i}$, where $A_{i}, B_{i}$ are fixed in $\mathcal{A}$, is referred to as an elementary operator on $\mathcal{A}$. If $A, B \in \mathcal{A}$, we denote by $M_{A, B}$ the operator $T \mapsto A T B$. The operator $M_{A, B}$ is called a basic elementary operator or a two-sided multiplication operator. The literature related to (basic) elementary operators is by now very large, much of it in the setting of $C^{*}$-algebras or in the Banach space setting. In this direction there are many excellent surveys and expositions. See, e.g., [4, 5, 6, 8,

For the study of two-sided multiplication operators in the setting of Riesz spaces (i.e., vector lattices) we would like to mention the work of Synnatzschke 9]. The set of all regular operators (order continuous regular operators, resp.) from a Riesz space $X$ into a Dedekind complete Riesz space $Y$ will be denoted by $L^{r}(X, Y)$ $\left(L_{n}^{r}(X, Y)\right.$, resp.). When $Y=\mathbb{R}$, we write $X^{\sim}$ and $X_{n}^{\sim}$ respectively instead of $L^{r}(X, \mathbb{R})$ and $L_{n}^{r}(X, \mathbb{R})$. They are likewise Dedekind complete Riesz spaces. Let $W, X, Y$ and $Z$ be Dedekind complete Riesz spaces. For all $A \in L^{r}(Y, Z)$ and $B \in L^{r}(W, X), M_{A, B}: T \in L^{r}(X, Y) \mapsto A T B \in L^{r}(W, Z)$ is a regular operator, and hence the modulus $\left|M_{A, B}\right|$ of $M_{A, B}$ exists in $L^{r}\left(L^{r}(X, Y), L^{r}(W, Z)\right)$. It is interesting to know about the relationship of $\left|M_{A, B}\right|$ with $|A|$ and $|B|$. Among other things, Synnatzschke [9, Satz 3.1] proved the following result:

a) If $0 \leq B_{0} \in L^{r}(W, X)$, then $\left|M_{A, B_{0}}\right|=M_{|A|, B_{0}}, M_{A, B_{0}} \vee M_{C, B_{0}}=M_{A \vee C, B_{0}}$ hold for all $A, C \in L^{r}(Y, Z)$.

b) If $0 \leq A_{0} \in L_{n}^{r}(Y, Z)$ and $Y_{n}^{\sim}, Z_{n}^{\sim}$ are total, then we have $\left|M_{A_{0}, B}\right|(T)=$ $M_{A_{0}, B \mid}(T)$ and $\left(M_{A_{0}, B} \vee M_{A_{0}, D}\right)(T)=M_{A_{0}, B \vee D}(T)$ for all $B, D \in L^{r}(W, X)$ and all $T \in L_{n}^{r}(X, Y)$.

2010 Mathematics Subject Classification. Primary 46A40; Secondary 46B42, 47B65.

Key words and phrases. regular operator, two-sided multiplication operator, Riesz space, Banach lattice.

The first author was supported in part by China Scholarship Council (CSC) and was visiting the University of South Carolina when this work was completed. 
Hereby $Y_{n}^{\sim}$ is total if $Y_{n}^{\sim}$ separates the points of $Y$. Synnatzschke uesd (a) to establish (b) by taking adjoints of operators. For his purpose, the hypothesis of both $Y_{n}^{\sim}$ and $Z_{n}^{\sim}$ being total is essential.

Recently, Wickstead [10] showed that if $E$ is an atomic Banach lattice with order continuous norm and $A, B \in L^{r}(E)$, then $\left|M_{A, B}\right|=M_{|A|,|B|}$ and $\left\|M_{A, B}\right\|_{r}=$ $\|A\|_{r}\|B\|_{r}$. In his proofs he depended heavily upon the 'atomic' condition.

In this note, we generalize the related results of Synnatzschke and Wickstead, respectively. We remove the condition of order continuous duals being total in [9, Satz 3.1(b)] and show that for every $0 \leq A_{0} \in L_{n}^{r}(Y, Z),\left|M_{A_{0}, B}\right|(T)=M_{A_{0},|B|}(T)$ holds for all $B \in L^{r}(W, X)$ and all $T \in L_{n}^{r}(X, Y)$. Furthermore, if $W, X, Y$ and $Z$ are Dedekind complete Banach lattices such that $X$ and $Y$ have order continuous norms (not necessarily atomic), then $\left|M_{A, B}\right|=M_{|A|,|B|}$ and $\left\|M_{A, B}\right\|_{r}=\|A\|_{r}\|B\|_{r}$ hold for all $A \in L^{r}(Y, Z)$ and all $B \in L^{r}(W, X)$.

Our notions are standard. For the theory of Riesz spaces and regular operators, we refer the reader to the monographs [2, 1, 11].

\section{The modulus of THE TWO-SIDED MULtiplication OPERATOR}

We start with two examples which serve to illustrate that the order continuous dual $X_{n}^{\sim}$ of a Dedekind complete Riesz space $X$ need not be total. This justifies our effort to generalize the result of Synnatzschke [9, Satz 3.1 b)].

Example 2.1. (1) Let $(\Omega, \Sigma, \mu)$ be a nonatomic finite measure space. Then the Dedekind complete Riesz space $X=L_{p}(\Omega, \Sigma, \mu)(0<p<1)$ satisfies $X_{n}^{\sim}=X^{\sim}=$ $\{0\}$. This result is due to M. M. Day. (cf. [3, Theorem 5.24, p. 128]).

(2) Let $K$ be a compact Hausdorff space. It is well known that $C(K)$ is Dedekind complete if and only if $K$ is Stonian (i.e., extremally disconnected). A Hausdorff compact Stonian space $K$ such that $C(K)_{n}^{\sim}$ is total is called hyper-Stonian. Dixmier gave a characterization of hyper-Stonian spaces: $K$ is hyper-Stonian if and only if $C(K)$ is isomorphic to a dual Banach lattice (cf. [7, Theorem 2.1.7]). He also gave a Dedekind complete $C(K)$-space which is not isomorphic to a dual space (see, e.g., [1, p. 99, Problems 4.8 and 4.9] for details). That is, such a $C(K)$ is Dedekind complete, but $C(K)_{n}^{\sim}$ is not total.

Proposition 2.2. Let $W, X, Y$ and $Z$ be Riesz spaces with $X, Y$ and $Z$ Dedekind complete. Let $0 \leq A_{0} \in L_{n}^{r}(Y, Z)$. Then we have $\left|M_{A_{0}, B}\right|(T)=M_{A_{0}, B \mid}(T)$ and, equivalently, $M_{A_{0}, B} \vee M_{A_{0}, D}(T)=M_{A_{0}, B \vee D}(T)$ for all $B, D \in L^{r}(W, X)$ and all $T \in L_{n}^{r}(X, Y)$.

Proof. For $B \in L^{r}(W, X)$ and $0 \leq T \in L_{n}^{r}(X, Y)$, we have to prove that $\left|M_{A_{0}, B}\right|(T)=$ $M_{A_{0},|B|}(T)$. Clearly we have $\left|M_{A_{0}, B}\right|(T) \leq M_{A_{0},|B|}(T)$, since $\left|M_{A_{0}, B}\right| \leq M_{A_{0}, B \mid}$ holds in $L^{r}\left(L^{r}(X, Y), L^{r}(W, Z)\right)$. For the reverse inequality, let $w \in W^{+}$. By a formula for the modulus of regular operators in [2, Theorem 1.21(3)] or [11, Theorem 20.10(i)] we have

$$
\left(\sum_{i=1}^{n}\left|B w_{i}\right|: n \in \mathbb{N}, 0 \leq w_{i} \in W, \sum_{i} w_{i}=w\right) \uparrow|B| w .
$$


Since $A_{0}$ and $T$ are both positive order continuous operators, $A_{0} T$ is likewise an order continuous positive operator from $X$ into $Z$. It follows that

$$
\begin{aligned}
M_{A_{0},|B|}(T)(w) & =A_{0} T|B| w \\
& =\sup \left(\sum_{i=1}^{n} A_{0} T\left|B w_{i}\right|: n \in \mathbb{N}, 0 \leq w_{i} \in W, \sum_{i} w_{i}=w\right)
\end{aligned}
$$

For each $1 \leq i \leq n$, let $P_{i}$ be the order projection from $X$ onto the band generated by $\left(B w_{i}\right)^{+}$in $X$ and let $Q_{i}=P_{i}-I$, where $I$ is the identity operator on $X$. Clearly,

$$
P_{i} \perp Q_{i}, \quad\left|P_{i}\right|+\left|Q_{i}\right|=I, \quad P_{i} B w_{i}=\left(B w_{i}\right)^{+}, \quad\left(P_{i}+Q_{i}\right) B w_{i}=\left|B w_{i}\right|,
$$

and

$$
\left|T P_{i}\right|+\left|T Q_{i}\right|=T
$$

Therefore, for each $i$ we have

$$
\begin{aligned}
A_{0} T\left|B w_{i}\right| & =\left(A_{0} T P_{i}+A_{0} T Q_{i}\right) B w_{i} \\
& \leq\left(\left|A_{0}\left(T P_{i}\right) B\right|+\left|A_{0}\left(T Q_{i}\right) B\right|\right) w_{i} \\
& \leq\left(\sup \left\{\sum_{j=1}^{m}\left|A_{0} T_{j} B\right|: m \in \mathbb{N}, T_{j} \in L^{r}(X, Y), \sum_{j}\left|T_{j}\right|=T\right\}\right) w_{i} \\
& =\left(\sup \left\{\sum_{j=1}^{m}\left|M_{A_{0}, B}\left(T_{j}\right)\right|: m \in \mathbb{N}, T_{j} \in L^{r}(X, Y), \sum_{j}\left|T_{j}\right|=T\right\}\right) w_{i} \\
& =\left|M_{A_{0}, B}\right|(T)\left(w_{i}\right) .
\end{aligned}
$$

Hence, from this it follows that

$$
\begin{aligned}
M_{A_{0},|B|}(T)(w) & =\sup \left(\sum_{i=1}^{n} A_{0} T\left|B w_{i}\right|: n \in \mathbb{N}, 0 \leq w_{i} \in W, \sum_{i} w_{i}=w\right) \\
& \leq \sup \left(\sum_{i=1}^{n}\left|M_{A_{0}, B}\right|(T)\left(w_{i}\right): n \in \mathbb{N}, 0 \leq w_{i} \in W, \sum_{i} w_{i}=w\right) \\
& =\left|M_{A_{0}, B}\right|(T)(w),
\end{aligned}
$$

which implies that $\left|M_{A_{0}, B}\right|(T) \leq M_{A_{0},|B|}(T)$ for all $B \in L^{r}(W, X)$ and all $0 \leq T \in$ $L_{n}^{r}(X, Y)$.

In general we can not expect that $\left|M_{A_{0}, B}\right|=M_{A_{0}, B \mid}$ holds for all $B \in L^{r}(W, X)$. That is, the linear operator $M_{A_{0}, .}: B \in L^{r}(W, X) \rightarrow L^{r}\left(L^{r}(X, Y), L^{r}(W, Z)\right)$ is not necessarily a Riesz homomorphism. In the last section we give a counterexample to illustrate this. However, for Banach lattices with order continuous norms the situation is quite different. The next result is a consequence of the above proposition and the earlier result of Synnatzschke [9, Satz 3.1], which generalizes Theorem 3.1 of Wickstead [10] recently obtained for atomic Banach lattices with order continuous norms.

Corollary 2.3. Let $W, X, Y$ and $Z$ be Banach lattices such that $X, Y$ have order continuous norms and $Z$ is Dedekind complete. Then we have $\left|M_{A, B}\right|=M_{|A|,|B|}$ for all $A \in L^{r}(Y, Z)$ and all $B \in L^{r}(W, X)$. 
Proof. Let $\mathcal{M}: L^{r}(Y, Z) \times L^{r}(W, X) \rightarrow L^{r}\left(L^{r}(X, Y), L^{r}(W, Z)\right)$ be the bilinear operator defined via $\mathcal{M}(A, B)=M_{A, B}$. Clearly, $\mathcal{M}$ is positive. Since $X$ and $Y$ are Banach lattices with order continuous norms, we have $L_{n}^{r}(X, Y)=L^{r}(X, Y)$ and $L_{n}^{r}(Y, Z)=L^{r}(Y, Z)$. From Proposition 2.2 above and the result of Synnatzschke [9, Satz 3.1] it follows that for every $0 \leq A_{0} \in L^{r}(Y, Z)$ and every $0 \leq B_{0} \in L^{r}(W, X)$, $\mathcal{M}\left(A_{0}, \cdot\right)$ and $\mathcal{M}\left(\cdot, B_{0}\right)$ are both Riesz homomorphisms. Hence, for all $A \in L^{r}(Y, Z)$ and all $B \in L^{r}(W, X)$ we have

$$
\begin{aligned}
\left|M_{A, B}\right| & =|\mathcal{M}(A, B)| \\
& =\left|\mathcal{M}\left(A^{+}-A^{-}, B^{+}-B^{-}\right)\right| \\
& =\left|\mathcal{M}\left(A^{+}, B^{+}\right)-\mathcal{M}\left(A^{+}, B^{-}\right)-\mathcal{M}\left(A^{-}, B^{+}\right)+\mathcal{M}\left(A^{-}, B^{-}\right)\right| \\
& =\mathcal{M}\left(A^{+}, B^{+}\right)+\mathcal{M}\left(A^{+}, B^{-}\right)+\mathcal{M}\left(A^{-}, B^{+}\right)+\mathcal{M}\left(A^{-}, B^{-}\right) \\
& =\mathcal{M}(|A|,|B|)=M_{|A|,|B|} .
\end{aligned}
$$

Here we are using the fact that the terms $\mathcal{M}\left(A^{+}, B^{+}\right), \mathcal{M}\left(A^{+}, B^{-}\right), \mathcal{M}\left(A^{-}, B^{+}\right)$ and $\mathcal{M}\left(A^{-}, B^{-}\right)$are pairwise disjoint.

Let $W$ and $X$ be Banach lattices with $X$ Dedekind complete. Recall that $L^{r}(W, X)$ is a Dedekind complete Banach lattice under the regular norm $\|B\|_{r}:=\||B|\|$ for every $B \in L^{r}(W, X)$. Note that $M_{A, B}$ is a regular operator from $L^{r}(X, Y)$ into $L^{r}(W, Z)$. The following result deals with the regular norms of two-sided multiplication operators. Its proof is based on Corollary 2.3

Corollary 2.4. If $W, X, Y$ and $Z$ be Banach lattices such that $X, Y$ have order continuous norms and $Z$ is Dedekind complete, then $\left\|M_{A, B}\right\|_{r}=\|A\|_{r}\|B\|_{r}$ for all $A \in L^{r}(Y, Z)$ and all $B \in L^{r}(W, X)$.

Proof. We first assume that $0 \leq A \in L^{r}(Y, Z)$ and $0 \leq B \in L^{r}(W, X)$. Since $M_{A, B} \geq 0$, we have $\left\|M_{A, B}\right\|_{r}=\left\|M_{A, B}\right\| \leq\|A\|\|B\|=\|A\|_{r}\|B\|_{r}$. On the other hand, for every $0 \leq x^{\prime} \in X^{\prime}$ and every $0 \leq y \in Y$ satisfying $\left\|x^{\prime}\right\| \leq 1$ and $\|y\| \leq 1$, $x^{\prime} \otimes y \in L^{r}(X, Y)$ and $\left\|x^{\prime} \otimes y\right\|_{r}=\left\|x^{\prime} \otimes y\right\| \leq 1$. Then it follows that

$$
\begin{aligned}
\left\|M_{A, B}\right\|_{r}=\left\|M_{A, B}\right\| & \geq \sup \left(\left\|M_{A, B}\left(x^{\prime} \otimes y\right)\right\|: 0 \leq x^{\prime} \in B_{X^{\prime}}, 0 \leq y \in B_{Y}\right) \\
& =\sup \left(\left\|\left(B^{\prime} x^{\prime}\right) \otimes A y\right\|: 0 \leq x^{\prime} \in B_{X^{\prime}}, 0 \leq y \in B_{Y}\right) \\
& =\|A\|\|B\|=\|A\|_{r}\|B\|_{r} .
\end{aligned}
$$

This implies that $\left\|M_{A, B}\right\|_{r}=\|A\|_{r}\|B\|_{r}$ holds for all $0 \leq A \in L^{r}(Y, Z)$ and $0 \leq B \in$ $L^{r}(W, X)$.

Now, for the general case let $A \in L^{r}(Y, Z)$ and $B \in L^{r}(W, X)$ be arbitrary. Then by Corollary 2.3 we have

$$
\left\|M_{A, B}\right\|_{r}=\left\|\left|M_{A, B}\right|\right\|=\left\|M_{|A|,|B|}\right\|=\left\|M_{|A|,|B|}\right\|_{r}=\|A\|_{r}\|B\|_{r} .
$$

Wickstead [10] establishes that even in the case of atomic Banach lattices with order continuous norms the operator norm of two-sided multiplication operators need not be equivalent to the regular norm. 
TWO-SIDED MULTIPLICATION OPERATORS ON THE SPACE OF REGULAR OPERATORS 5

\section{A Counterexample}

Let $X$ and $Y$ be Riesz spaces with $Y$ Dedekind complete. The set of all $\sigma$ order continuous operators in $L^{r}(X, Y)$ will be denoted by $L_{c}^{r}(X, Y)$. The disjoint complement $\left(L_{c}^{r}(X, Y)\right)^{d}$ of $L_{c}^{r}(X, Y)$ is denoted by $L_{s}^{r}(X, Y)$. Every element of $L_{s}^{r}(X, Y)$ is called a singular operator. When $Y=\mathbb{R}$, we write $X^{\sim}$ and $X_{s}^{\sim}$ respectively instead of $L^{r}(X, \mathbb{R})$ and $L_{s}^{r}(X, \mathbb{R})$. The following example illustrates that $\left|M_{A_{0}, B}\right|=M_{A_{0},|B|}$ does not necessarily hold for all $B \in L^{r}(W, X)$, that is, the linear operator $M_{A_{0}, .}: B \in L^{r}(W, X) \rightarrow L^{r}\left(L^{r}(X, Y), L^{r}(W, Z)\right)$ is not necessarily a Riesz homomorphism in general.

Example 3.1. Let $W=X=Y=Z=\ell_{\infty}$ and let $e$ denote the strong unit $(1,1, \cdots)$ of $\ell_{\infty}$. Let $0 \leq f \in\left(\ell_{\infty}\right)_{s}^{\sim}$ be a singular Riesz homomorphism with $f(e)=1$ (one can take, e.g., $f$ equal to a limit over a free ultrafilter). Let $B \in L^{r}\left(\ell_{\infty}\right)$ be the rank one operator $B=f \otimes e$. Then it is clear that $B \in L_{s}^{r}\left(\ell_{\infty}\right)$ and $I \wedge B=0$, where $I$ is the identity operator on $\ell_{\infty}$ (and hence order continuous). We claim that $M_{I, I} \wedge M_{I, B} \neq M_{I, I \wedge B}=0$. To this end, let $0 \leq T \in L^{r}\left(\ell_{\infty}\right)$. Then, by [2, Theorem $1.21(2)]$ we have

$$
\left\{\sum_{i=1}^{n}\left(T_{i} \wedge T_{i} B\right): n \in \mathbb{N}, T_{i} \geq 0, \sum_{i} T_{i}=T\right\} \downarrow\left(M_{I, I} \wedge M_{I, B}\right)(T) .
$$

From this and [2, Theorem 1.51(2)] it follows that

$$
\begin{aligned}
&\left(M_{I, I}\right.\left.\wedge M_{I, B}\right)(T)(e) \\
&=\inf \left\{\sum_{i=1}^{n}\left(T_{i} \wedge T_{i} B\right)(e): n \in \mathbb{N}, T_{i} \geq 0, \sum_{i} T_{i}=T\right\} \\
&=\inf \left\{\sum_{i=1}^{n}\left(T_{i} \wedge\left(f \otimes T_{i} e\right)\right)(e): n \in \mathbb{N}, T_{i} \geq 0, \sum_{i} T_{i}=T\right\} \\
&=\inf \left\{\sum_{i=1}^{n} \inf \left(\sum_{j=1}^{m_{i}} T_{i} x_{j}^{(i)} \wedge f\left(x_{j}^{(i)}\right) T_{i} e: x_{j}^{(i)} \wedge x_{k}^{(i)}=0, j \neq k, \sum_{j=1}^{m_{i}} x_{j}^{(i)}=e\right):\right. \\
&\left.n \in \mathbb{N}, T_{i} \geq 0, \sum_{i} T_{i}=T\right\} .
\end{aligned}
$$

Let us put

$$
\begin{array}{r}
G^{\prime}=\left\{\sum_{i=1}^{n} \inf \left(\sum_{j=1}^{m_{i}} T_{i} x_{j}^{(i)} \wedge f\left(x_{j}^{(i)}\right) T_{i} e: x_{j}^{(i)} \wedge x_{k}^{(i)}=0, j \neq k, \sum_{j=1}^{m_{i}} x_{j}^{(i)}=e\right):\right. \\
\left.n \in \mathbb{N}, T_{i} \geq 0, \sum_{i} T_{i}=T\right\}, \\
G^{\prime \prime}=\left\{\sum_{i=1}^{n} \sum_{j=1}^{m} T_{i} x_{j} \wedge f\left(x_{j}\right) T_{i} e: m \in \mathbb{N}, x_{j} \wedge x_{k}=0, j \neq k, \sum_{j=1}^{m} x_{j}=e,\right. \\
\left.n \in \mathbb{N}, T_{i} \geq 0, \sum_{i} T_{i}=T\right\} .
\end{array}
$$


We claim that $\inf G^{\prime}=\inf G^{\prime \prime}$. Indeed, it is clear that $\inf G^{\prime} \leq \inf G^{\prime \prime}$. For the reverse inequality, let $\left(T_{i}\right)_{1}^{n}$ be a fixed positive partition of $T$ (i.e., $T_{i} \geq 0, \sum_{i} T_{i}=$ $T)$. For each $i$ let $\left(x_{j}^{(i)}\right)_{j=1}^{m_{i}}$ be an arbitrary positive disjoint partition of $e$ (i.e., $x_{j}^{(i)} \wedge x_{k}^{(i)}=0, j \neq k, \sum_{j=1}^{m_{i}} x_{j}^{(i)}=e$ ) corresponding to $T_{i}$. Following the proof of [2, Theorem 1.51] we can find a positive disjoint partition $\left(x_{j}\right)_{1}^{m}$ of $e$ such that

$$
\sum_{j=1}^{m} T_{i} x_{j} \wedge f\left(x_{j}\right) T_{i} e \leq \sum_{j=1}^{m_{i}} T_{i} x_{j}^{(i)} \wedge f\left(x_{j}^{(i)}\right) T_{i} e \quad(i=1,2, \cdots, n) .
$$

From this it follows that

$$
\inf G^{\prime \prime} \leq \sum_{i=1}^{n} \sum_{j=1}^{m} T_{i} x_{j} \wedge f\left(x_{j}\right) T_{i} e \leq \sum_{i=1}^{n} \sum_{j=1}^{m_{i}} T_{i} x_{j}^{(i)} \wedge f\left(x_{j}^{(i)}\right) T_{i} e
$$

Therefore,

$$
\inf G^{\prime \prime} \leq \sum_{i=1}^{n} \inf \left(\sum_{j=1}^{m_{i}} T_{i} x_{j}^{(i)} \wedge f\left(x_{j}^{(i)}\right) T_{i} e: x_{j}^{(i)} \wedge x_{k}^{(i)}=0, j \neq k, \sum_{j=1}^{m_{i}} x_{j}^{(i)}=e\right),
$$

which implies that $\inf G^{\prime \prime} \leq \inf G^{\prime}$. Hence, we have $\left(M_{I, I} \wedge M_{I, B}\right)(T)(e)=\inf G^{\prime \prime}$.

Since $f$ is a Riesz homomorphism, for every positive disjoint partition $\left(x_{j}\right)_{1}^{m}$ of $e$ appearing in $G^{\prime \prime}$ there exists only one, say $x_{j_{0}}$, such that

$$
\begin{array}{r}
f\left(x_{j}\right)=0, \quad j \neq j_{0}, \quad f\left(x_{j_{0}}\right)=f(e)=1 \\
x_{j_{0}} \wedge \sum_{j \neq j_{0}} x_{j}=x_{j_{0}} \wedge\left(e-x_{j_{0}}\right)=0 .
\end{array}
$$

It follows that $\sum_{i=1}^{n} \sum_{j=1}^{m} T_{i} x_{j} \wedge f\left(x_{j}\right) T_{i} e=\sum_{i=1}^{n} T_{i} x_{j_{0}}$. On the other hand, for any $x \in E^{+}$satisfying $x \wedge(e-x)=0$ and $f(x)=1$, we must have $f(e-x)=0$, and hence

$$
\sum_{i=1}^{n} T_{i} x=\sum_{i=1}^{n}\left(T_{i} x \wedge f(x) T_{i} e+T_{i}(e-x) \wedge f(e-x) T_{i} e\right)
$$

Thus, we have

$$
\begin{aligned}
&\left(M_{I, I} \wedge\right.\left.M_{I, B}\right)(T)(e) \\
&= \inf \left\{\sum_{i=1}^{n} \sum_{j=1}^{m} T_{i} x_{j} \wedge f\left(x_{j}\right) T_{i} e: m \in \mathbb{N}, x_{j} \wedge x_{k}=0, j \neq k, \sum_{j=1}^{m} x_{j}=e,\right. \\
&\left.n \in \mathbb{N}, T_{i} \geq 0, \sum_{i} T_{i}=T\right\} \\
&=\inf \left\{\sum_{i=1}^{n} T_{i} x: x \wedge(e-x)=0, f(x)=1, n \in \mathbb{N}, T_{i} \geq 0, \sum_{i} T_{i}=T\right\} \\
&=\inf \{T x: 0 \leq x \leq e, x \wedge(e-x)=0, f(x)=1\} .
\end{aligned}
$$

If we now take $T=B=f \otimes e$, then $\left(M_{I, I} \wedge M_{I, B}\right)(f \otimes e)(e)=e$. So, $M_{I, I} \wedge M_{I, B} \neq 0$. 


\section{REFERENCES}

[1] F. Albiac and N. J. Kalton, Topics in Banach Space Theory, Graduate Texts in Mathematics 233, Springer, New York, 2006. MR2006h:46005

[2] C. D. Aliprantis and O. Burkinshaw, Positive Operators (reprint of the 1985 original), Springer, Dordrecht, 2006. MR2262133

[3] C. D. Aliprantis and O. Burkinshaw, Locally Solid Riesz Spaces with Applications to Economics, Math Surveys and Monographs, Volume $\sharp 105$, Amer. Math. Soc., 2003. MR2005b:46010.

[4] P. Ara and M. Mathieu, Local Multipliers of $C^{*}$-Algebras, Springer Monographs in Mathematics, Springer-Verlag, London, 2003. MR2008i:47076

[5] R. E. Curto, Spectral theory of elementary operators, Elementary operators and applications (M. Mathieu, ed.)(Proc. Int. Conf. Blaubeuren, 1991), 3-52, World Sci. Publ., River Edge, NJ, 1992. MR93i:47041

[6] M. Mathieu, The norm problem for elementary operators, Recent progress in functional analysis (Valencia, 2000), 363-368, North-Holland Math. Stud., 189, North-Holland, Amsterdam, 2001. MR2002g:47071

[7] P. Meyer-Nieberg, Banach Lattices, Universitext, Springer-Verlag, Berlin, 1991. MR 93f:46025

[8] E. Saksman and H.-O. Tylli, Multiplications and elementary operators in the Banach space setting, Methods in Banach space theory, 253-292, London Math. Soc. Lecture Note Ser., 337, Cambridge Univ. Press, Cambridge, 2006. MR2008i:47076

[9] J. Synnatzschke, Über einige verbandstheoretische Eigenschaften der Multiplikation von Operatoren in Vektorverbänden, Math. Nachr. 95 (1980), 273-292. MR82b:47048

[10] A. W. Wickstead, Norms of basic elementary operators on algebras of regular operators, Proc. Amer. Math. Soc., to appear.

[11] A. C. Zaanen, Introduction to Operator Theory in Riesz Spaces, Springer, Berlin, 1997. MR2000c:47074

Department of Mathematics, Southwest Jiaotong University, Chengdu 610031, PR CHINA

E-mail address: jinxichen@home.swjtu.edu.cn

Department of Mathematics, University of South Carolina, Columbia, SC 29208

E-mail address: schep@math.sc.edu 